\title{
Argon Impurity Transport Studies at Wendelstein 7-X using X-ray Imaging Spectrometer Measurements
}

\author{
A Langenberg ${ }^{1}$, N A Pablant ${ }^{2}$, O Marchuk ${ }^{3}$, D Zhang ${ }^{1}$, A Alonso ${ }^{4}$, \\ R Burhenn ${ }^{1}$, J Svensson ${ }^{1}$, P Valson ${ }^{1}$, D Gates ${ }^{2}$, M Beurskens ${ }^{1}$, R C Wolf ${ }^{1}$, and \\ the W7-X Team \\ ${ }^{1}$ Max-Planck-Institut für Plasmaphysik, Greifswald, Germany \\ ${ }^{2}$ Princeton Plasma Physics Laboratory, Princeton, NJ, USA \\ ${ }^{3}$ Institut für Energie und Klimaforschung, Forschungszentrum Jülich $\mathrm{GmbH}$, \\ Jülich, Germany \\ ${ }^{4}$ Laboratorio Nacional de Fusión - CIEMAT, 28040 Madrid, Spain \\ andreas.langenberg@ipp.mpg.de
}

\begin{abstract}
In the first operational phase of the stellarator Wendelstein 7-X (W7-X), the X-ray Imaging Crystal Spectrometer (XICS) system has been commissioned for measuring radial profiles of ion and electron temperature, $T_{i}$ and $T_{e}$, plasma rotation velocities $v_{P}$, and selected impurity densities $n_{Z}$. This paper shows the first measurements of the spectrometer and gives an initial calculation of impurity transport parameters derived from an Ar impurity transport study. Using Bayesian analysis, the temporal evolution of Ar impurity density profiles after an Ar gas puff could be observed with a time resolution of up to $5 \mathrm{~ms}$, yielding a maximum value for the diffusion coefficient of $D=1.5 \mathrm{~m}^{2} / \mathrm{s}$ at $\rho \sim 0.5$ and small pinch velocities in the inner plasma region.
\end{abstract}

PACS: 52.25.Fi, 52.25.Vy

\section{Introduction}

In the first operational phase of the stellarator W7-X, plasmas with peaked temperature profiles up to $T_{e}=8 \mathrm{keV}$ and $T_{i}=2 \mathrm{keV}$ and maximum pulse lengths of $6 \mathrm{~s}$ have been achieved [1,2]. Moreover, experiment programs of stationary ion and electron temperature and electron density profiles, $n_{e}$, have been established, enabling the conduction of initial impurity transport experiments by puffing Ar gas into the plasma [3]. Here, Ar densities have been monitored using an x-ray imaging crystal spectrometer system XICS, which is a standard diagnostic for the determination of radial profiles of $T_{i}$ and $T_{e}$, plasma rotation velocities $v_{P}$, and selected impurity densities $n_{Z}[4-8]$.

On the way towards a steady state operation of $\mathrm{W} 7-\mathrm{X}$, the investigation of impurity transport is of particular interest. For stellarators, neoclassical theory predicts a general impurity inward 
drift which may lead to impurity accumulation in the plasma center and, in the worst case, to a potential plasma collapse due to impurity radiation, especially for long pulse times in the order of several minutes. Therefore, plasma scenarios preventing or minimizing impurity accumulation needs to be developed, using dedicated transport studies.

Impurity transport experiments typically consist of injecting impurity species into the plasma and monitoring its temporal and/or radial distribution, using several diagnostics. A comparison of measured impurity abundances with simulated ones using various transport codes yields estimations on diffusive and convective transport coefficients [9-18]. Alternatively, fluxes and transport coefficients can be derived from the knowledge of total impurity densities, as shown for impurity transport [19-20] and also for electron transport [21], neglecting external sink and source terms. However, for one particular charge state, it is not straight forward to measure transport coefficients and impurity fluxes directly, since e.g. $\mathrm{x}$-ray based measurements using soft $\mathrm{x}$-ray diodes average over a sum of multiple charge states.

In this paper, a method for the determination of transport coefficients directly from imaging spectrometer measurements is shown, as to our knowledge, for the first time. The method relies on the measurement of neighbouring impurity charge states that together with its temporal evolution fully define the flux of one particular charge state. The particular interest on a transport study using high resolution $\mathrm{x}$-ray spectra is also stimulated by the ability of these diagnostics to distinguish between different charge states. Here, calculations are done for $\mathrm{Ar}^{16+}$ (He-like argon), yielding initial observations of core transport coefficients in W7-X.

\section{XICS Spectrometer Design}

The X-ray Imaging Crystal Spectrometer XICS has been set up at W7-X in collaboration with the Princeton Plasma Physics Laboratory [22]. The spectrometer is equipped with a spherical bent crystal, so that x-rays emitted from the plasma are imaged onto a two dimensional detector with energy resolution in horizontal, and spatial resolution in vertical direction on the detector. Figure 1 a) shows the designed viewing geometry of XICS with a spatial range covering radial positions $\rho=0.92$ above the magnetic axis and $\rho=0.41$ below the magnetic axis. Throughout this paper, $\rho$ denotes the square root of the magnetic flux $\psi$ normalized to the magnetic flux of the last closed flux surface $\psi_{L C F S}$ with the magnetic axis at $\rho=0$ and the 
last closed flux surface at $\rho=1.0$. Depending on the crystal choice and set Bragg angle, spectra of selected impurities, e.g. Ar, of a particular charge state can be monitored. Typical raw images for the emission of $\operatorname{Ar}^{16+}$ ( $w, x, y$, and $z$ lines) are shown in Figure $1 \mathrm{~b}$ ), measured for the Bragg angle of $54.0^{\circ}$ using a quartz crystal.

\section{Temperature and Density Profile Inference}

A spectral fit of line of sight integrated spectra and a tomographic inversion of XICS data yields profiles of the above mentioned plasma parameters as shown in figure 2. Spectral fits (figure 2(a), green lines) of measured data (figure 2(a), blue lines) show a reasonably good match with deviations on the order of the photon statistics. In figure $2 \mathrm{~b}$ ), measured and fitted line intensities of selected emission lines are shown. Due to the asymmetric flux surface geometry, the line of sight integrated brightness profiles above the magnetic axis (filled symbols) differ from views below the magnetic axis (open symbols). From Doppler broadening and line intensity ratios of the emission lines, line of sight integrated $T_{i}$ and $T_{e}$ values (blue and red dots in figure $2 \mathrm{c}$ ) can be determined directly from the spectral fit.

The inference of actual temperature and density profiles has been done in the Minerva framework [23], with an entire forward model of XICS using Bayesian analysis for error estimation [24]. Thin gray solid lines in figure 2 c) and d) represent sampled profiles from the error distribution which yield forward modeled XICS data matching measured data within the photon statistics, the displayed error bars are the standard deviation of sampled profiles. The simultaneous fit of all emission lines in the spectra, including dielectronic satellites, recombination of $n_{A r}{ }^{17+}$, and excitation of $n_{A r}{ }^{15+}$, allows to infer $n_{A r}{ }^{15+}, n_{A r}{ }^{16+}$, and $n_{A r}{ }^{17+}$ profiles (figure $2 \mathrm{~d}$, green, red, and orange lines). While e.g. the (line of sight integrated) $n_{A r}{ }^{15+}$ density relates directly to the $q$ satellite intensity, $n_{A r}{ }^{16+}$, and $n_{A r}{ }^{17+}$ densities are not directly given but result from a complex mixture of emission lines with different dependencies on the main excitation $w, x, y, z$, and several satellite lines. For details, see $[25,26]$. Effects of charge exchange with the neutral $\mathrm{H}$ background gas have been neglected. We note the increase of the ratio between the $\mathrm{z}$ and the $\mathrm{w}$ line at the plasma edge. It is a strong indicator on the presence of neutrals [27] accompanied by transport processes driving $\mathrm{H}$-like Ar towards the plasma edge $[28,29]$ so that the diagnostic can be potentially used to derive the neutral density profile at the plasma edge. For instance, the increased ratio between $n_{A r}{ }^{17+}$ to $n_{A r}{ }^{16+}$ (figure $2 \mathrm{~d}$ ) towards the edge shows the role of the charge-exchange recombination in 
He-like spectra. All Ar densities are given in arbitrary units as an absolute density calibration is not available yet. However, the relative scaling of different charge states is accurate. The electron temperature and electron density profiles have been taken from Thomson scattering data (dashed lines in figure $2 \mathrm{c}+\mathrm{d}$ ).

According to the fractional abundances of charge states at different $T_{e}, \mathrm{Ar}^{17+}$ is expected to be the dominant charge state for $T_{e}>3 \mathrm{keV}$, followed by $\mathrm{Ar}^{16+}$ and a small contribution from $\mathrm{Ar}^{15+}$. Moreover, the penetration depth into the plasma center of $n_{A r}{ }^{17+}$ and $n_{A r}{ }^{16+}$ is significantly larger compared to $n_{A r}^{15+}$, see figure $2 \mathrm{~d}$ ).

For a first estimate of expected $n_{A r}$ profiles, figure 3 a) shows fractional abundances of Ar in the coronal limit. Obviously, measured Ar profiles do clearly deviate from the coronal limit profiles, pointing out the influence of additional transport effects. To illustrate this point, a STRAHL [30,31] transport code analysis has been carried out. Making use of measured $T_{e}$ and $n_{e}$ profiles and a defined Ar gas puff outside the last closed flux surface, the temporal evolution of Ar charge states has been calculated in a 1D model, parameterizing relevant quantities like emissivity $\varepsilon, n_{Z}$, and both diffusive and convective transport coefficients $D$ and $v$ as functions of the effective radius $r$, defined as $r=\rho a$, with the minor radius $a=0.49 \mathrm{~m}$ as defined by the limiters. For the STRAHL simulations, plasma edge and recycling properties have been taking into account by setting the following parameters [30]: Recycling of Ar from the limiter has been considered by using recycling factors $R \sim 1$, yielding a preferably match of simulated signals to measured ones. The pumping efficiency has been ignored by setting the pumping time constant to a very large value, based on the fact that the pumping power during the first experimental phase of W7-X has been low. A scrape-off layer with a thickness of $8.0 \mathrm{~cm}$ is used according to the edge diagnostic information. The decay length $\lambda$ of the impurity outside the last grid point has been set to $\lambda=1 \mathrm{~cm}$, the radial flux density at the last mesh point is $\Gamma_{\mathrm{z}}=D_{\text {edge }} n_{z \text {,edge }} / \lambda$. For the Ar gas puff, a realistic time dependent Ar puff rate with the source of Ar $10 \mathrm{~cm}$ away from the last closed flux surface and an energy of neutral Ar of $1 \mathrm{eV}$ has been assumed. Reference [30] describes how STRAHL treats the transport in the scrape off layer and which numerical algorithms are used.

In figure $3 \mathrm{~b}$ ), the resulting Ar density profiles at $t=500 \mathrm{~ms}$, assuming a constant diffusion coefficient $\left(D=0.5 \mathrm{~m}^{2} / \mathrm{s}\right)$ and zero ion drift velocity as a first approach, are shown. The inclusion of transport effects significantly improved the overall match of simulated and measured Ar profile shapes (compare figure $2 \mathrm{~d}$ ) and $3 \mathrm{~b}$ ), which shows that before mentioned deviations from the coronal equilibrium can be attributed to the radial transport of the 
impurity species. However, the peaking of $\mathrm{n}_{\mathrm{Ar}}{ }^{15+}$ and $\mathrm{n}_{\mathrm{Ar}}{ }^{16+}$ profiles at $r=0.35-0.4$ in the simulation is much less pronounced for the measured profiles. This is an indication for a larger diffusion coefficient for those radial positions. In the next section, an alternative method for the determination of impurity fluxes and transport coefficients is discussed in detail.

\section{Argon Impurity Transport Study}

For a more detailed analysis of impurity transport, Ar gas puff experiments have been performed. Therefore, a small amount of Ar gas has been puffed for $30 \mathrm{~ms}$ in non-perturbing trace amounts $\left(n_{A r}<5 \times 10^{-15} \mathrm{~m}^{-3}\right)$ into the plasma at a stationary phase of static $T_{e}, T_{i}$, and $n_{e}$ profiles, typically $150 \mathrm{~ms}$ after the start of the ECR heating. In figure $4 \mathrm{a}$ )-d), time traces of the ECR heating power, the central $T_{e}, T_{i}$, and $n_{e}$ are shown together with the onset of the Ar gas puff. While the central $T_{e}$ and the heating power can be assumed to be stationary, central $T_{i}$ and $n_{e}$ still show a slight temporal evolution after the Ar puff for $t>150 \mathrm{~ms}$, respectively. Since the $n_{e}$ profile is an input parameter used for the spectral fit of XICS spectra, the measured $n_{e}$ evolution has been taken into account for each time step of the Ar profile inference. The $T_{i}$ profile has been set as a free parameter and inferred together with the $\mathrm{Ar}$ density profiles, as shown exemplary for $t=230 \mathrm{~ms}$ in figure $2 \mathrm{c}$ ). In figure $4 \mathrm{e}$ ), time traces of Ar densities $n_{A r}{ }^{15+}, n_{A r}{ }^{16+}$, and $n_{A r}{ }^{17+}$ normalized to one are shown. One observes a clear temporal delay of emission between different ionization stages. In particular, the Li-like $\mathrm{Ar}^{15+}$ emission peaks at $t=0.20-0.22 \mathrm{~s}$, the maximum emission of $\mathrm{Ar}^{16+}$ occurs at $t=0.22-0.24 \mathrm{~s}$, and finally the maximum of the $\mathrm{Ar}^{17+}$ emission is achieved slightly later at $\mathrm{t}=0.23-0.25 \mathrm{~s}$ as indicated by the arrows in figure $4 \mathrm{e}$ ). The time delay on the order of $15 \mathrm{~ms}$ is consistent with the ionization time $\tau$ of different ionization stages $\tau_{i}=1 /\left(S \cdot n_{e}\right)$, with the ionization rate $S$. Assuming an electron temperature of $5 \mathrm{keV}\left(S=6 \cdot 10^{-12} \mathrm{~cm}^{3} / \mathrm{s}\right)$ and a plasma density of $1.5 \cdot 10^{19} \mathrm{~m}^{3}$ yields an estimation of the $\mathrm{Ar}^{16+}$ ionization time of $11 \mathrm{~ms}$, being in a good agreement with the time delay observed between the $\mathrm{Ar}^{16+}$ and $\mathrm{Ar}^{17+}$ ionization stages in figure $4 \mathrm{e})$.

Figure 5 a)-c) shows the measured time dependent evolution of $n_{A r}{ }^{15+}, n_{A r}^{16+}$, and $n_{A r}{ }^{17+}$ profiles with a $5 \mathrm{~ms}$ time resolution for the experiment program 160310.028. Starting at 185 ms (35 ms after the Ar gas puff), a pronounced and fast rise of the $\mathrm{Ar}^{16+}$ density profiles with 
a rise time of $30 \pm 5 \mathrm{~ms}$ can be observed, followed by a slow decay phase. A similar behavior can be seen for the $n_{A r}{ }^{15+}$ and $n_{A r}{ }^{17+}$ profiles, see also figure $4 \mathrm{e}$ ).

The measured temporal evolution of $n_{A r}{ }^{15+}, n_{A r}{ }^{16+}$, and $n_{A r}{ }^{17+}$ profiles averaged over the magnetic flux surface are now used to calculate the Ar flux $\Gamma_{A r}{ }^{16+}$ of the particular charge state $\mathrm{Ar}^{16+}$ as follows: By integrating the continuity equation

$$
\frac{\partial n_{A r^{16+}}}{\partial t}=-\frac{1}{r} \frac{\partial}{\partial r}\left(r \Gamma_{A r^{16+}}\right)+Q
$$

over the effective radius $r$ one gets for the flux of $\mathrm{Ar}^{16+}$ the following expression:

$$
\Gamma_{A r^{16+}}(r)=-\frac{1}{r} \int_{0}^{r} \frac{\partial n_{A r^{16+}}}{\partial t} r^{\prime} d r^{\prime}+\frac{1}{r} \int_{0}^{r} Q r^{\prime} d r^{\prime}
$$

where $\partial n_{A r}{ }^{16+} / \partial t$ is the time derivative of the $n_{A r}{ }^{16+}$ density. Sinks and sources of $n_{A r}{ }^{16+}$ are determined by recombination and ionization processes:

$$
Q=n_{e}\left(S_{A r^{15+}} n_{A r^{15+}}-S_{A r^{16+}} n_{A r^{16+}}-R_{A r^{16+}} n_{A r^{16+}}+R_{A r^{17+}} n_{A r^{17+}}\right)
$$

The ionization $S$ and recombination rate coefficients $R$ for all impurity charge states are taken from the ADAS database [32]. Note, that with the above defined effective radius using the square root of normalized magnetic flux $\left(r=a \rho=a \sqrt{ } \psi / \psi_{L C F S}\right)$, equations 1 and 2 can be applied to axisymmetric as well as non-axisymmetric plasmas. For high-aspect-ratio systems like stellarators, the specific volume $\partial V / \partial \psi$ varies only slightly with radius. Its radial variation relative to its central value is the so-called magnetic well which is typically in the $1 \%$ range. Therefore, $r$ defined using the toroidal flux $r \sim \sqrt{ } \psi / \psi_{\text {LCFS }}$ is almost identical to the often used definition of $r$ based on the volume $V$ of flux surfaces $r \sim \sqrt{ } V / V_{L C F S}$.

The flux $\Gamma$ associated to the time derivative of $n_{A r}{ }^{16+}$ profiles (left side of Eq.2) is shown in figure $5 \mathrm{~d}$ ). Right after the Ar gas puff, $\Gamma$ profiles are negative until the $n_{A r}{ }^{16+}$ profiles saturate, reflecting the penetration of $\mathrm{Ar}^{16+}$ into the plasma. For $t>230 \mathrm{~ms}$, the $n_{A r}{ }^{16+}$ density 
slowly decays, resulting in slightly positive $\Gamma$ flux profiles. Sink and source profiles (figure 5 e) basically resemble the shapes of the Ar density profiles, with $n_{A r}{ }^{16+}$ source terms $(Q>0)$ for ionization of $\mathrm{Ar}^{15+}$ and recombination of $\mathrm{Ar}^{17+}$ (green and orange profiles), and sinks $(Q<$ 0 ) for ionization and recombination of $\mathrm{Ar}^{16+}$ (red profiles).

The corresponding total $\mathrm{Ar}^{16+}$ flux $\Gamma_{A r}{ }^{16+}$ do have a pronounced time dependence, especially in the transient phase of the Ar influx. This allows an estimation of the diffusion and convection parameters under the assumption that they remain constant in time according to a simple linear model:

$$
\Gamma_{A r^{16+}}(r)=-D \frac{\partial n_{A r^{16+}}}{\partial r}+v n_{A r^{16+}}
$$

For a determination of $D$ and $v$, the dynamic evolution of Ar density profiles can be used: After the Ar gas puff, $\mathrm{Ar}^{16+}$ fluxes and density gradients, $\Gamma_{A r}{ }^{16+}$ and $\partial n_{A r}{ }^{16+} / \partial r$, evolve in time, mapping $\mathrm{Ar}^{16+}$ fluxes for different density gradients. A normalization of equation 4 through $n_{A r}{ }^{16+}$ and plotting normalized flux $\Gamma_{A r}{ }^{16+} / n_{A r}{ }^{16+}$ versus normalized density gradients $\partial n / \partial r /$ $n_{A r}{ }^{16+}$ allows the determination of $D$ and $v$ from the slope and the offset of a linear fit for the corresponding radial positions $r$ as shown in figure $5 \mathrm{f}$ ).

Figure 6 shows the resulting $D$ and $v$ profiles for the $\mathrm{Ar}^{16+}$ charge state. The diffusion coefficient $D$ rises from the plasma center towards larger plasma radii to a value of $D=(1.5 \pm$ $0.8) \mathrm{m}^{2} / \mathrm{s}$ at $\rho=0.45$. Drift velocities $v$ are close to zero with uncertainties of about $\pm 2 \mathrm{~m} / \mathrm{s}$. The statistics of inferred Ar profiles are too low for reasonable estimations of $D$ at $\rho>0.6$ and $v$ at $\rho>0.4$. However, inferred $D$ and $v$ profiles are consistent with transport coefficients used for the above shown 1D STRAHL transport calculation, where constant profiles of $D=0.5$ $\mathrm{m}^{2} / \mathrm{s}$ and $v=0 \mathrm{~m} / \mathrm{s}$ have been assumed (figure $3 \mathrm{~b}$ ) for simplicity. Deviations of $\mathrm{D}$ and $\mathrm{v}$ profiles shown in figure 6 compared to those assumed for the STRAHL simulation can be attributed to the fact that no optimization of STRAHL simulations to measured data has been done so far.

Improved statistics of measured XICS data and reduced uncertainties of inferred Ar profiles would allow a determination of the entire $D$ and $v$ profiles with much higher accuracies. For that purpose, several options like increasing the amount of puffed Ar in transport studies, binning of more spatial lines of sight, or a reduction of the time resolution are available and have not been optimized yet. 


\section{Summary}

In this paper, an initial observation of the impurity fluxes and transport coefficients $D$ and $v$ of the $\mathrm{Ar}^{16+}$ impurity charge state based on XICS measurements has been presented. One observed He-like spectra of argon at different vertical positions at W7-X. For the spectral fitting and tomographic inversion of recorded data, the Minerva Bayesian analysis framework has been used. The general behavior of the line emission could be well described within the atomic model used in combination with the transport code STRAHL. The temporal evolution of the Li-like, He-like, and H-like charge states could be detected and is consistent with a simple estimation of the ionization time of particular ionization states. Moreover, the spectra were used to derive first data on the impurity transport properties of the W7-X plasma. In the plasma core, a zone of reduced transport is observed, increasing towards the edge with maximum $D=1.5 \mathrm{~m}^{2} / \mathrm{s}$ at $\rho=0.5$. The impact of the charge exchange and $n_{H}$ recombination on the spectra was still neglected which prevents to extrapolate data towards $\rho>0.5$ and conclusions on the density of neutral hydrogen atoms. In the near future the atomic model is going to be improved. In addition, the derived $D$ and $v$ profiles are consistent with assumed transport coefficients of a 1D STRAHL transport code simulation.

\section{Acknowledgments}

This work has been carried out within the framework of the EUROfusion Consortium and has received funding from the Euratom research and training programme 2014-2018 under grant agreement No 633053. The views and opinions expressed herein do not necessarily reflect those of the European Commission.

\section{References}

[1] Klinger T et al. 2017 Plasma Phys. Control. Fusion 59014018

[2] Wolf R C et al. Major results from the first plasma campaign of the Wendelstein 7-X stellarator, submitted to Nucl. Fusion

[3] Langenberg A et al. $201643^{\text {rd }}$ EPS Conference on Plasma Physics (Leuven) P4.014

[4] Reinke M L et al. 2012 Rev. Sci. Instrum. 83113504

[5] Shi Y et al. 2010 Plasma Phys. Control. Fusion 52085014

[6] Ince-Cushman A et al. 2008 Rev. Sci. Instrum. $7910 \mathrm{E} 302$ 
[7] Lee S G, Yoo J W, Kim Y S, Nam U W and Moon M K 2016 Rev. Sci. Instrum. 87 $11 \mathrm{E} 314$

[8] Pablant N A et al. 2012 Rev. Sci. Instrum. 83083506

[9] Zhang K et al. 2016 Chin. Phys. B 25065202

[10] Arevalo J, Alonso J A, McCarthy K J and Velasco J L 2013 Nucl. Fusion 53023003

[11] Menmuir S, Carraro L, Alfier A, Bonomo F, Fassina A, Spizzo G and

Vianello N 2010 Plasma Phys. Control. Fusion 52095001

[12] Leigheb M et al. 2007 Plasma Phys. Control. Fusion 49 1897-1912

[13] Marchuk O et al. 2006 Plasma Phys. Control. Fusion 481633

[14] Burhenn R et al. 2004 Fusion Sci. Technol. 46115

[15] Tamura N et al. 2003 Plasma Phys. Control. Fusion 45 27-41

[16] Delgado-Aparicio L et al. 2009 Nucl. Fusion 49085028

[17] Puiatti M E et al. 2002 Plasma Phys. Control. Fusion 44 1863-1878

[18] Dux R, Peeters A G, Gude A, Kallenbach A, Neu R and the ASDEX Upgrade Team 1999 Nucl. Fusion 391509

[19] Sertoli M, Angioni C, Dux R, Neu R, Putterich T, Igochine V and

the ASDEX Upgrade Team 2011 Plasma Phys. Control. Fusion 53035024

[20] Ida K et al. 2009 Phys. Plasmas 16056111

[21] Guirlet R et al. 2010 Nucl. Fusion 50095009

[22] Pablant N et al. $201643^{\text {rd }}$ EPS Conference on Plasma Physics (Leuven) P4.013

[23] Svensson J, Werner A 2007 IEEE International Symposium on Intelligent Signal

Processing

[24] Langenberg A, Svensson J, Thomsen H, Marchuk O, Pablant N A, Burhenn R and

Wolf R C 2016 Fusion Sci. Technol. 69560

[25] Schlummer T 2014 Charge exchange recombination in X-ray spectra of He-like argon measured at the tokamak TEXTOR PhD thesis, University Düsseldorf

[26] Marchuk O 2004 Modeling of He-like spectra measured at the tokamaks TEXTOR and TORE SUPRA $\mathrm{PhD}$ thesis, University Bochum

[27] Kato T, Masai K, Fujimoto T, Koike F, Källne E, Marmar E S and Rice J E 1991 Phys. Rev. A, 446776

[28] Rice J E, Terry J L, Marmar E S and Bombarda F 1997 Nucl. Fusion 37241

[29] Reinke M L, Rice J E, Hutchinson I H, Greenwald M, Howard N T, Hughes J W, Irby J, Podpaly Y, Terry J L and White A 2013 Nucl. Fusion 53043006

[30] Dux R et al. 2006 IPP report 10/30

[31] Behringer K 1987 JET Report JET-R (87) 08 Joint European Torus

[32] http://open.adas.ac.uk/ 
a) Viewing geometry XICS

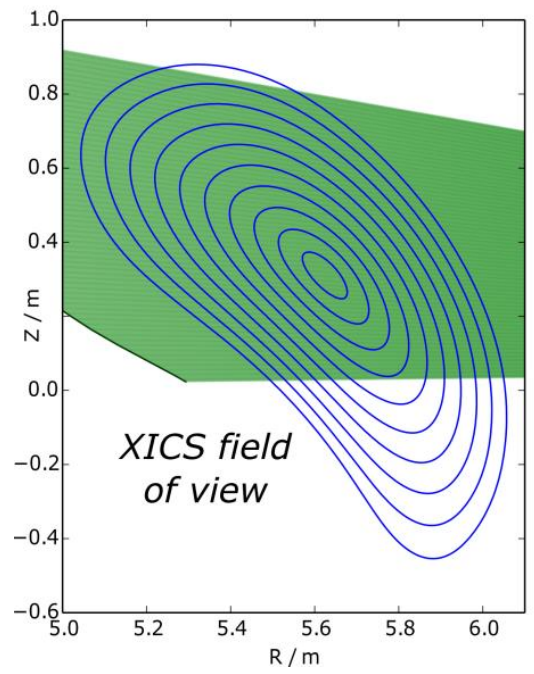

b) Detector Image

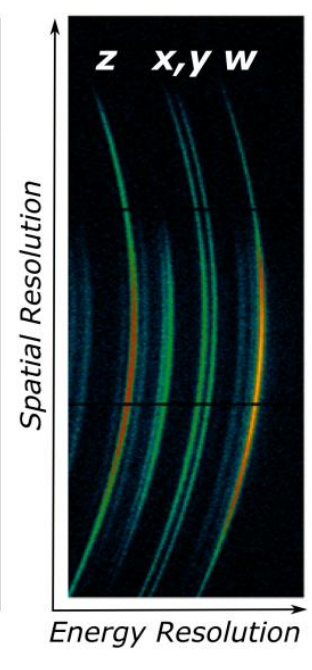

Figure 1: a) Viewing geometry of the XICS diagnostic (shaded area) with respect to flux surfaces (solid lines) shown for $\rho=0.1-1.0 . b$ ) Raw XICS detector image of $\mathrm{Ar}^{16+}$ emission. 
a)

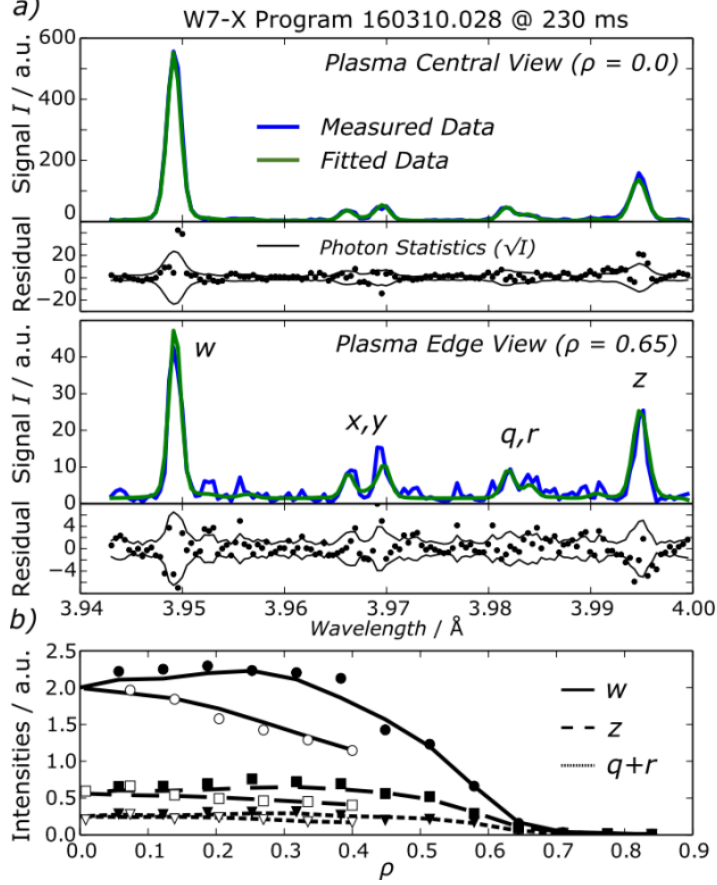

c)
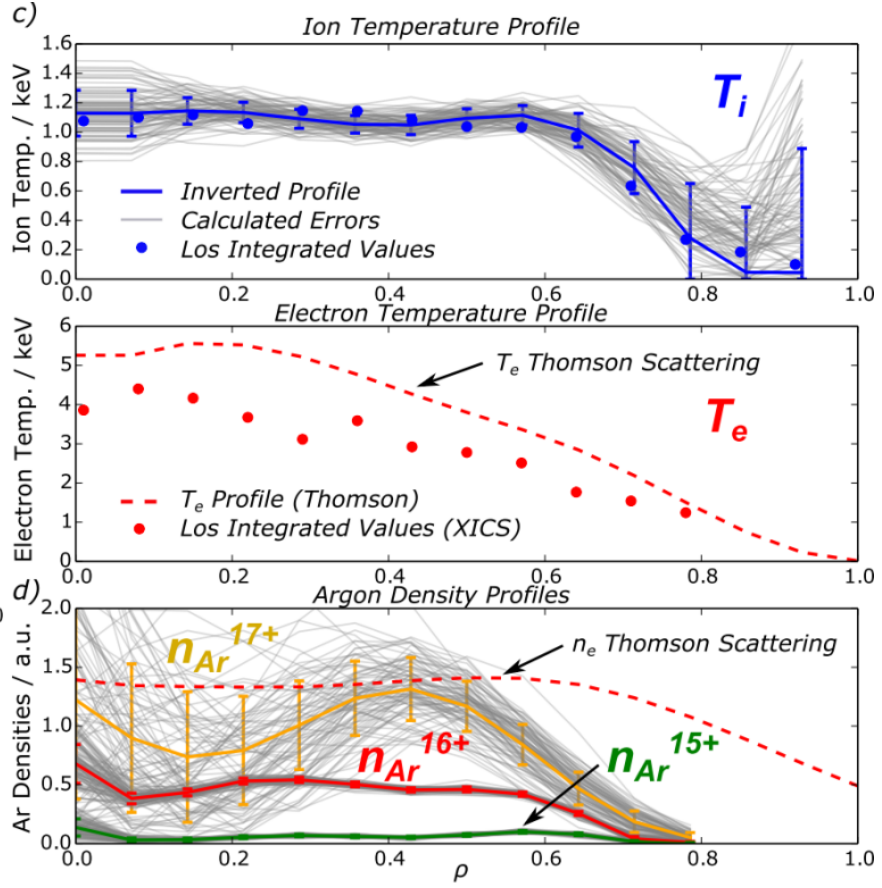

Figure 2: a) Measured and fitted XICS Ar ${ }^{16+}$ spectra along a central $(\rho=0)$ and a non central $(\rho=$ 0.65) line of sight. Residuals of the spectral fits are shown for each pixel (dots) together with photon statistics (solid lines). b) Measured (symbols) and fitted (lines) line intensities for $w, z$, and $q+r A r^{16+}$ emission lines above (filled symbols) and below (open symbols) the magnetic axis. c) Inferred ion temperature (bold solid line) profiles. Line of sight integrated temperature values are shown as dots. d) Inferred Ar density profiles of different charge states (bold orange, red, and green lines) including calculated errors and profiles sampled from the error distribution (thin solid lines). 
a) Ar Fractional Abundances

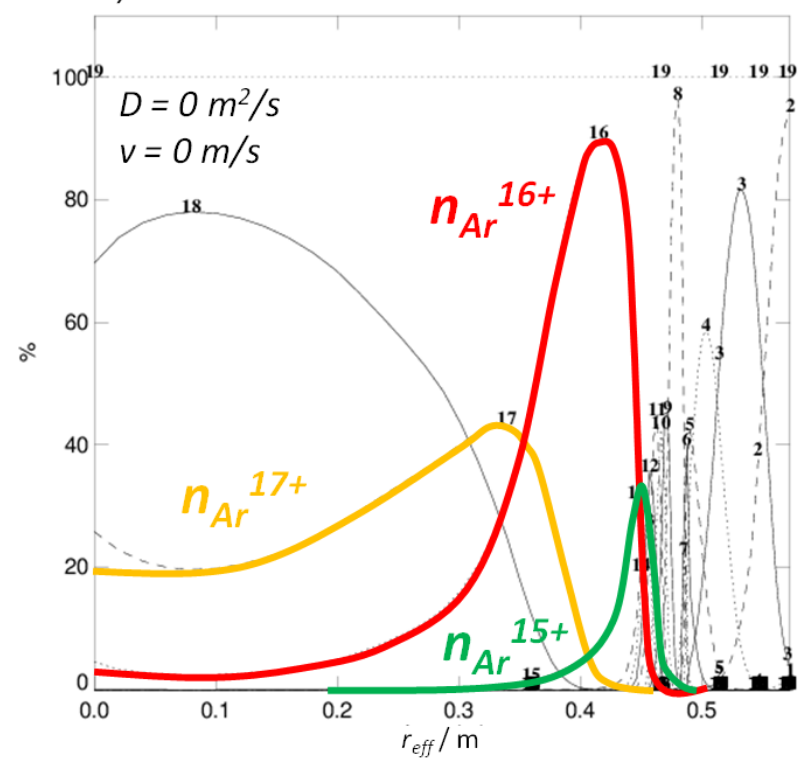

b) Ar Densities

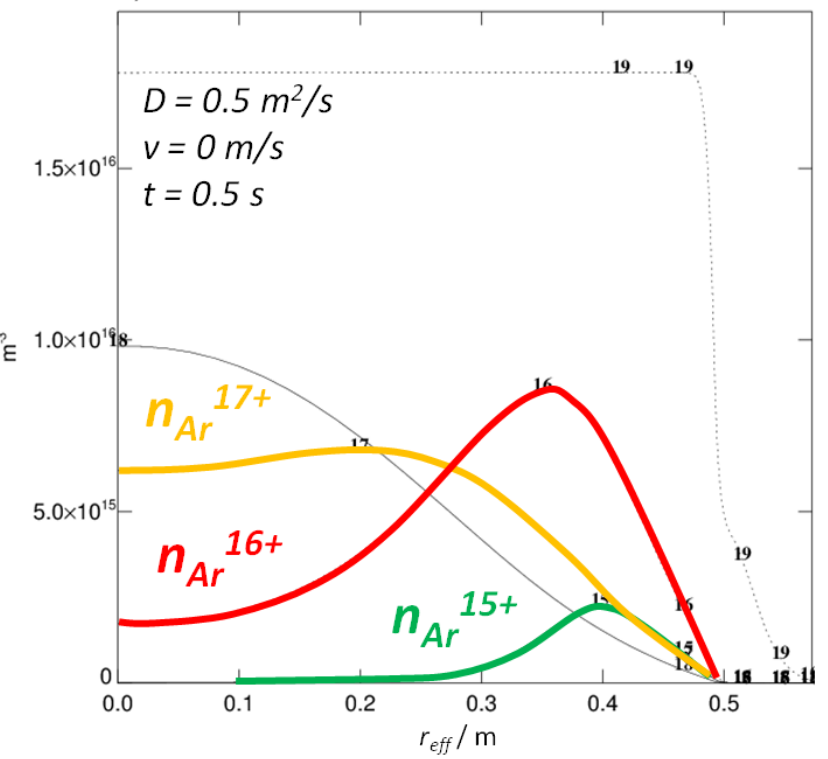

Figure 3: a) Fractional abundances of Ar for measured $T_{e}$ profile. b) Simulated Ar densities using a ID STRAHL transport code. 

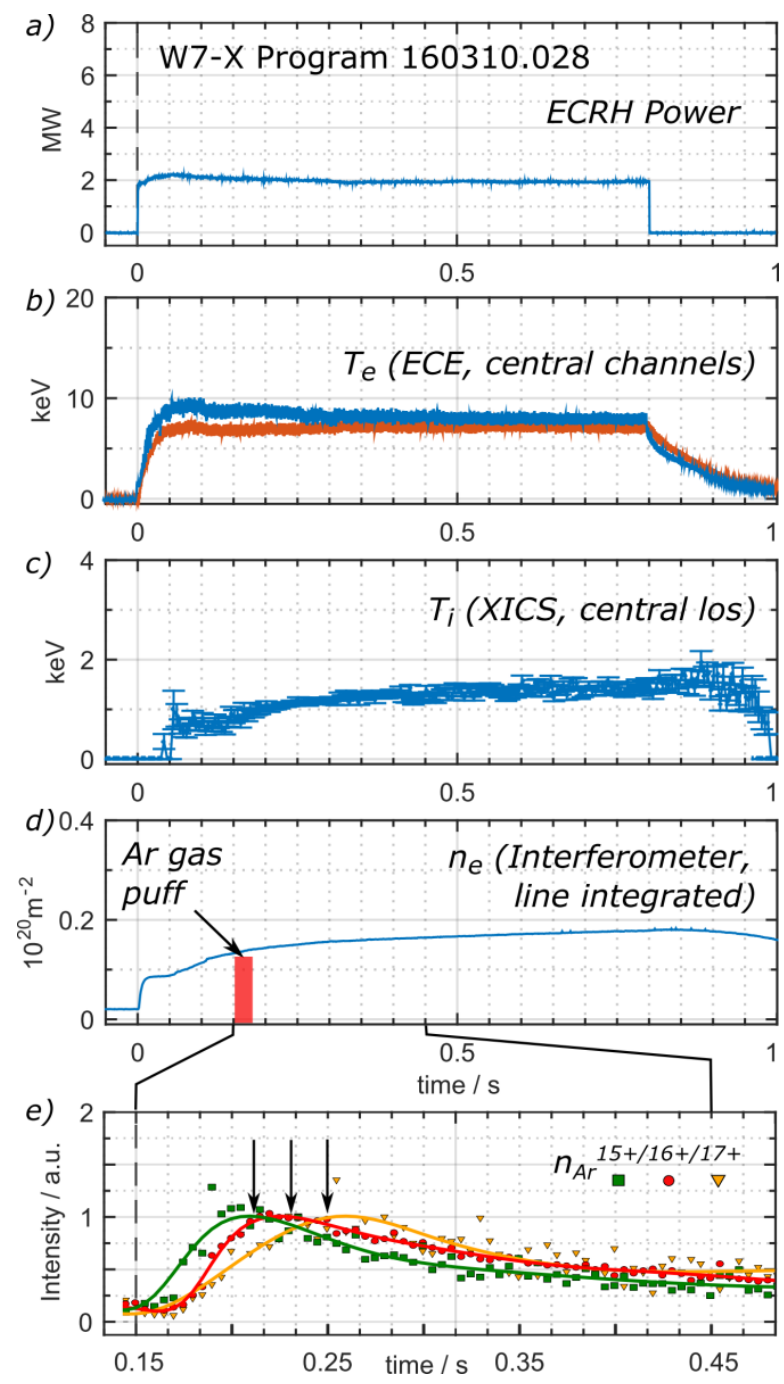

Figure 4: a)-e) Time traces of electron cyclotron resonance heating (ECRH) power, central $T_{e}$ from the electron cyclotron emission (ECE) diagnostic, central $T_{i}$ from XICS, central electron density $n_{e}$ from the interferometer diagnostic, and normalized Ar densities of $n_{A r}{ }^{15+}, n_{A r}{ }^{16+}$, and $n_{A r}{ }^{17+}$ for the program 160310.028. 
a)

nAr15+ Profiles

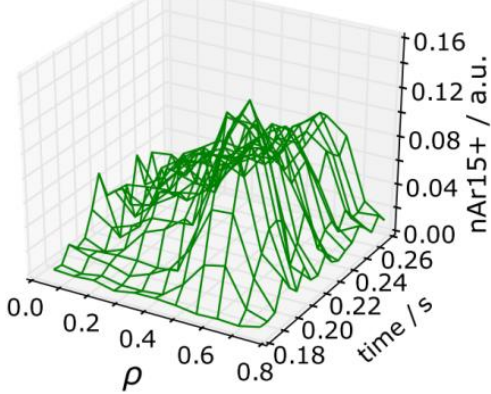

d)

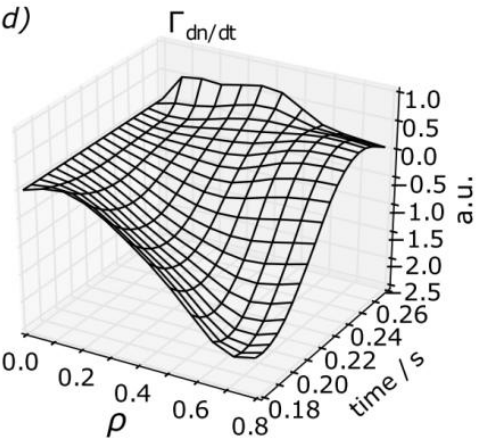

b)

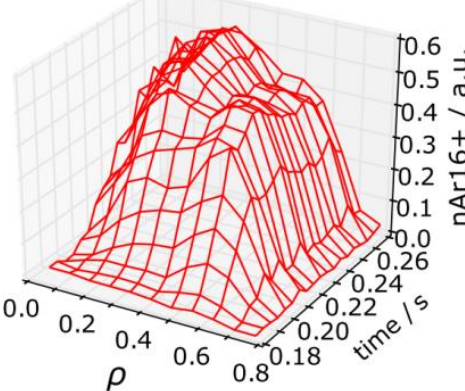

c) $\mathrm{nAr} 17+$ Profiles

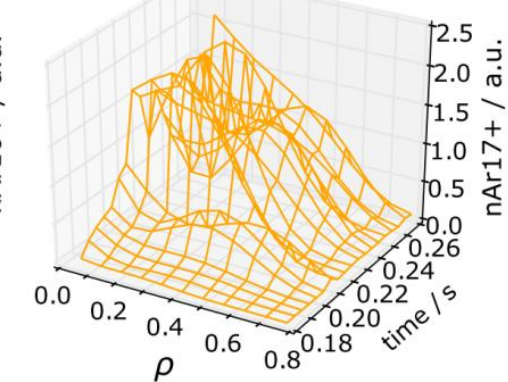

$\Gamma / \mathrm{n}$ vs $\operatorname{grad} \mathrm{n} / \mathrm{n}$

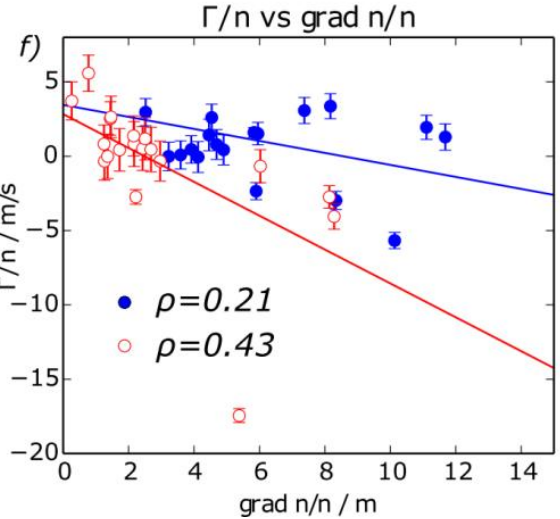

Figure 5: a)-c) Temporal evolution of measured $n_{A r}{ }^{15+}, n_{A r}{ }^{16+}$, and $n_{A r}{ }^{17+}$ density profiles after an Ar gas puff. d) Temporal evolution of flux profiles of $A r^{16+}$ (neglecting sinks and sources). e) Sinks and sources for $\mathrm{Ar}^{16+}$ (see text). f) Normalized Ar ${ }^{16+}$ fluxes plotted versus normalized density gradients for radial positions $\rho=0.21$ and 0.43 . 

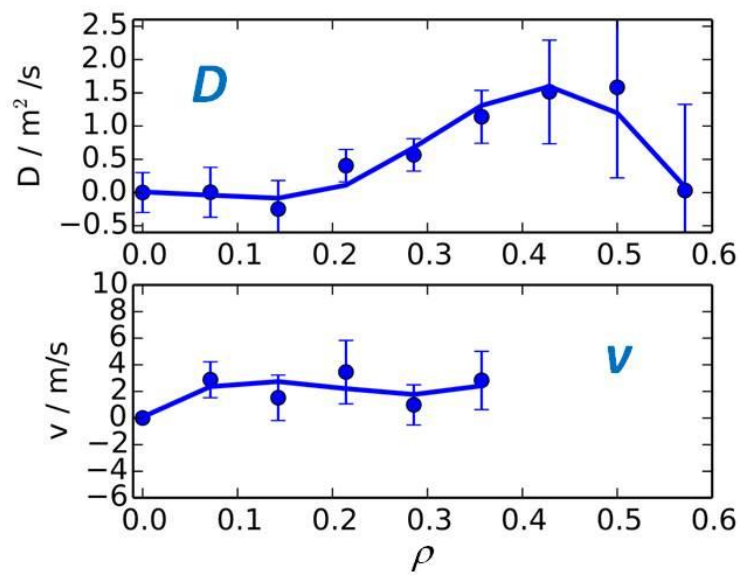

Figure 6: Inferred diffusive D and convective $v$ transport coefficients for the $A r^{16+}$ transport. 